\title{
On the Integrality Ratio for Tree Augmentation
}

\author{
J. Cheriyan * $\quad$ H. Karloff ${ }^{\dagger} \quad$ R. Khandekar ${ }^{\ddagger}$ Jochen Könemann ${ }^{\S}$
}

\begin{abstract}
We show that the standard linear programming relaxation for the tree augmentation problem in undirected graphs has an integrality ratio that approaches $\frac{3}{2}$. This refutes a conjecture of Cheriyan, Jordán, and Ravi (ESA 1999) that the integrality ratio is $\frac{4}{3}$.
\end{abstract}

Keywords: connectivity augmentation, 2-edge-connected graph, linear programming, integer programming, integrality ratio, tree augmentation.

\section{Introduction}

We study a standard linear programming (LP) relaxation of the TreE Augmentation problem, defined as follows: given an undirected graph $G=(V, E)$ with nonnegative costs on the edges, and a spanning tree $T=\left(V, E_{T}\right)$ of $G$, find a set $F^{*}$ of edges, $F^{*} \subseteq E \backslash E_{T}$, of minimum cost such that the graph $\left(V, E_{T} \cup F^{*}\right)$ is 2-edge connected. This is a special case of the well-known 2-EDGEConnected Spanning Subgraph (2ECSS) problem, in which we are given an undirected graph $G=(V, E)$ with nonnegative costs on the edges and the goal is to find a 2-edge-connected spanning subgraph of minimum cost, that is, find a set $E^{*} \subseteq E$ of minimum cost such that $\left(V, E^{*}\right)$ is 2-edge connected. The special case of tree augmentation arises when the edges of cost zero form a connected spanning subgraph. The 2ECSS problem, in turn, is a special case of the SuRvivable NeTwOrK DESIGN (SNDP) problem: we are given an undirected graph $G=(V, E)$ with nonnegative costs on the edges and an integer $r_{i, j} \geq 0$ for each unordered pair of nodes $i, j$, and the goal is to find a subgraph of minimum cost that has at least $r_{i, j}$ edge-disjoint paths between every pair of nodes $i, j$.

Consider a standard integer programming (IP) formulation of the tree augmentation problem. There is a (nonnegative) integer variable $x_{f}$ for each $f \in E \backslash E_{T}$. Observe that $E \backslash E_{T}$ is the set of nontree edges of $G$, and each $f \in E \backslash E_{T}$ corresponds to a path in the tree, namely, the path in $T$ between the two endpoints of $f$; we denote this path by $p(f)$. We say that $f \in E \backslash E_{T}$ covers an edge $e \in T$ if $(T \backslash\{e\}) \cup\{f\}$ is connected, that is, if $e \in p(f)$. For each edge $e$ of $T$, there is a constraint

$$
\sum_{f \in E \backslash E_{T}: e \in p(f)} x_{f} \geq 1
$$

\footnotetext{
*Dept. of Comb. \& Opt., U. Waterloo, Waterloo ON Canada N2L 3G1.

${ }^{\dagger}$ AT\&T Labs-Research, 180 Park Ave., Florham Park, NJ 07932, USA.

Email: jcheriyan@uwaterloo.ca.

${ }^{\ddagger}$ IBM T.J.Watson Research Center, Yorktown Heights, NY 10598, USA.

Email: howard@research.att.com.

${ }^{\S}$ Corresponding author.

Email: rkhandekar@gmail.com.

Email: jochen@uwaterloo.ca.

Dept. of Comb. \& Opt., U. Waterloo, Waterloo ON Canada N2L 3G1.
} 
where $\left\{f \in E \backslash E_{T}: e \in p(f)\right\}$ is the set of nontree edges of $G$ that cover $e$. The objective function is to minimize $\sum_{f \in E \backslash E_{T}} c_{f} x_{f}$, where $c_{f}$ denotes the cost of the nontree edge $f$. We obtain the following linear program by relaxing the integrality constraints on the variables:

$$
\begin{array}{ll}
\min & \sum_{\substack{f \in E \backslash E_{T} \\
\text { s.t. }}} c_{f} x_{f} \\
& \sum_{\substack{f \in E \backslash E_{T}: \\
e \in p(f)}} x_{f} \geq 1 \quad \forall e \in E_{T} \\
& x_{f} \geq 0 \quad \forall f \in E \backslash E_{T} .
\end{array}
$$

Clearly, $\left(\mathrm{P}_{0}\right)$ is solvable in polynomial time, since both the number of variables and the number of constraints are at most $|E|$.

The integrality ratio of $\left(\mathrm{P}_{0}\right)$ for a given instance is the ratio of the optimal cost of the integer program (IP) to the optimal cost of $\left(\mathrm{P}_{0}\right)$, assuming that the optima exist and the denominator is nonzero. The integrality ratio (or integrality gap) $\mathrm{IR}_{0}$ of $\left(\mathrm{P}_{0}\right)$ is the supremum of the integrality ratio over all instances of the problem. We use the same term in two different senses - one refers to an instance, the other to all instances - but the context will resolve the ambiguity. Jain's 2approximation algorithm for the survivable network design problem (SNDP) [6] implies that $\mathrm{IR}_{0}$ is at most two. As far as we know, the best lower bound known on $\operatorname{IR}_{0}$ was $\frac{4}{3}$ : Let $G$ be the complete graph on four nodes, let the tree $T$ consist of three edges incident to any one node, and let the three nontree edges have unit cost; then an optimal integral solution consists of two nontree edges, and an optimal solution to $\left(\mathrm{P}_{0}\right)$ assigns a value of $\frac{1}{2}$ to all three nontree edges. In fact, Cheriyan, Jordán, and Ravi conjectured [1, 1-cover conjecture] that $\frac{4}{3}$ was also an upper bound on $\mathrm{IR}_{0}$. Our main contribution is a family of instances in which all edge costs are unit and the integrality ratio approaches $\frac{3}{2}$; more precisely, for each $k \geq 1$, there is an instance defined by a graph $G_{k}$ on $2+2 k$ nodes that has integrality ratio at least $\frac{k+1}{2 k / 3+1}$.

The construction presented in this paper easily extends to several generalizations of the tree augmentation problem. Consider for example the following two linear programming formulations for the 2ECSS and SNDP problems, respectively. For a set $\emptyset \neq S \subset V$, we let $\delta(S)$ denote the set of edges with exactly one endpoint in $S$, and we use $\hat{r}(S)$ for $\max \left\{r_{i j} \mid i \in S, j \notin S\right\}$.

$$
\begin{aligned}
& \min \sum_{e \in E} c_{e} x_{e} \quad\left(\mathrm{P}_{1}\right) \quad \min \sum_{e \in E} c_{e} x_{e} \\
& \text { s.t. } \sum_{e \in \delta(S)} x_{e} \geq 2 \quad \forall \emptyset \neq S \subset V \quad \text { s.t. } \sum_{e \in \delta(S)} x_{e} \geq \hat{r}(S) \quad \forall \emptyset \neq S \subset V \\
& 0 \leq x_{e} \leq 1 \quad \forall e \in E \quad 0 \leq x_{e} \leq 1 \quad \forall e \in E
\end{aligned}
$$

A slight adaptation of the instance family presented in this paper gives a lower-bound of $\frac{3}{2}$ on the integrality ratio of $\left(\mathrm{P}_{1}\right)$. It is well-known that $\left(\mathrm{P}_{2}\right)$ has an integrality ratio of 2 for SNDP; however, this lower-bound does not apply to the special case of SNDP where $r_{i j} \in\{0,2\}$ for all $i, j$. In this case, our construction implies that $\left(\mathrm{P}_{2}\right)$ has an integrality ratio of $\frac{3}{2}$.

We note that the above relaxation for 2ECSS coincides with the well-known Held-Karp relaxation $[4,5]$ of the traveling salesman problem whenever edge-costs satisfy the triangle inequality 


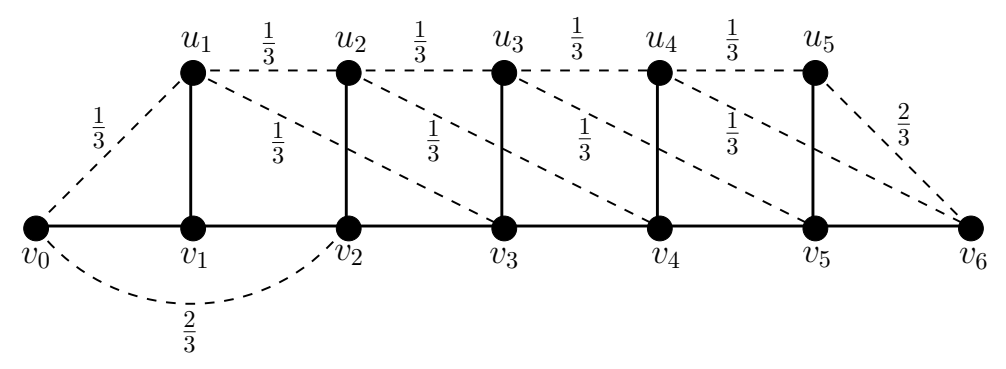

Figure 1: The integrality ratio example $G_{k}$ for $k=5$. The solid edges represent the tree edges and the dotted edges represent the nontree edges. All the nontree edges have unit cost. The fractions represent a feasible solution to $\left(\mathrm{P}_{0}\right)$ with cost $\frac{2 k}{3}+1$ while the integral optimal solution has cost $k+1$.

(see $[3,7]$ ). Our family of 2ECSS instances are not metric, however, and our results have no direct implication for the integrality ratio of the Held-Karp relaxation.

\section{The Construction}

Let $k \geq 1$ be any integer. We present an instance of the tree augmentation problem that has integrality ratio at least $\frac{k+1}{2 k / 3+1}$. The graph $G_{k}$ used in this instance has $2 k+2$ nodes $v_{0}, \ldots, v_{k+1}$, and $u_{1}, \ldots, u_{k}$. The edge set of $G_{k}$ has tree and nontree edges. The set of tree edges is given by

$$
\left\{\left\{v_{i}, v_{i+1}\right\} \mid 0 \leq i \leq k\right\} \cup\left\{\left\{u_{i}, v_{i}\right\} \mid 1 \leq i \leq k\right\},
$$

and we use $T_{k}$ to denote the spanning tree of $G_{k}$ induced by these edges. The nontree edges are $\left\{v_{0}, u_{1}\right\},\left\{v_{0}, v_{2}\right\} ;\left\{u_{i}, v_{i+2}\right\}$ for $1 \leq i \leq k-1 ;\left\{u_{i}, u_{i+1}\right\}$ for $1 \leq i \leq k-1$; and $\left\{u_{k}, v_{k+1}\right\}$. Each nontree edge has unit cost.

Consider the fractional solution $x^{k}$ depicted in Figure 1. It assigns a value of $\frac{2}{3}$ to $\left\{v_{0}, v_{2}\right\}$ and $\left\{u_{k}, v_{k+1}\right\}$ and a value of $\frac{1}{3}$ to all the other nontree edges. The cost of $x^{k}$ is $\frac{2 k}{3}+1$. To see that this solution is feasible for $\left(\mathrm{P}_{0}\right)$, it suffices to show that each tree edge is covered to an extent of at least one. This is true for edges $\left\{u_{i}, v_{i}\right\}$ for $1 \leq i \leq k-1$ as $u_{i}$ is incident to three nontree edges. Similarly, for all $2 \leq i \leq k-1$, a tree edge $\left\{v_{i}, v_{i+1}\right\}$ is covered by the three nontree edges $\left\{u_{i-1}, v_{i+1}\right\},\left\{u_{i}, v_{i+2}\right\}$, and $\left\{u_{i}, u_{i+1}\right\}$. The constraints for the remaining four tree edges are easily verified.

Proposition 1. The cost of the optimal integral solution in $G_{k}$ is $k+1$.

Proof. The proof is by induction on $k$. It is easy to see that the cost of an optimal solution in $G_{1}$ is 2 . We now consider the case where $k \geq 2$.

We first argue that there is an integral optimal solution $I_{k}$ in $G_{k}$ that contains the edge $\left\{u_{k}, v_{k+1}\right\}$. Any solution that does not contain this edge, must contain both $\left\{u_{k-1}, u_{k}\right\}$ and $\left\{u_{k-1}, v_{k+1}\right\}$ in order to cover $\left\{u_{k}, v_{k}\right\}$ and $\left\{v_{k}, v_{k+1}\right\}$, respectively. In such a case, we can replace edge $\left\{u_{k-1}, v_{k+1}\right\}$ with $\left\{u_{k}, v_{k+1}\right\}$ in $I_{k}$ and obtain another feasible solution with the same (optimal) cost. 
Assume now that $I_{k}$ is an optimum solution to $G_{k}$ that contains edge $\left\{u_{k}, v_{k+1}\right\}$. Contracting the edges along the cycle $\left\langle u_{k}, v_{k}, v_{k+1}, u_{k}\right\rangle$ creates the instance $G_{k-1}$. It can be seen that $I_{k} \backslash\left\{\left\{u_{k}, v_{k+1}\right\}\right\}$ is a feasible solution for $G_{k-1}$.

By the inductive hypothesis, any feasible integral solution in $G_{k-1}$ has cost at least $k$. Hence, $I_{k} \backslash\left\{\left\{u_{k}, v_{k+1}\right\}\right\}$ has cardinality at least $k$, and this implies that $I_{k}$ has at least $k+1$ edges.

Finally, the feasible integral solution $\left\{\left\{v_{0}, u_{1}\right\},\left\{u_{1}, u_{2}\right\}, \ldots,\left\{u_{k-1}, u_{k}\right\},\left\{u_{k}, v_{k+1}\right\}\right\}$ has cost $k+$ 1. Thus the proof is complete.

Thus the integrality ratio of the instance $G_{k}$ is at least $\frac{k+1}{2 k / 3+1}$. Since this ratio approaches $\frac{3}{2}$ as $k$ approaches infinity, it follows that $\mathrm{IR}_{0}$ is at least $\frac{3}{2}$. The fractional solution $x^{k}$ is neither optimal nor basic for $\left(\mathrm{P}_{0}\right)$, but our asymptotic bound of $\frac{3}{2}$ on the integrality ratio is tight for the family $\left\{G_{k} \mid k \geq 1\right\}$ of instances.

\section{Acknowledgment}

This research was partly funded by NSERC research grants OGP0138432 and OGP0288340.

\section{References}

[1] J. Cheriyan, T. Jordán, and R. Ravi. On 2-coverings and 2-packings of laminar families. In Proceedings, European Symposium on Algorithms, pages 510-520, 1999. A longer version is on the web: http://www.math.uwaterloo.ca/ jcheriyan/publications.html.

[2] G. N. Frederickson and J. Ja'Ja'. Approximation algorithm algorithms for several graph augmentation problems. SIAM J. Comput., 10(2):270-283, 1981.

[3] M. X. Goemans and D. Bertsimas. Survivable networks, linear programming relaxations and the parsimonious property. Math. Programming, 60:145-166, 1993.

[4] M. Held and R. M. Karp. The traveling-salesman and minimum cost spanning trees. Operations Research, 18:1138-1162, 1970.

[5] M. Held and R. M. Karp. The traveling-salesman problem and minimum spanning trees: part II. Math. Programming, 1:6-25, 1971.

[6] K. Jain. A factor 2 approximation algorithm for the generalized steiner network problem. Combinatorica, 21(1):39-60, 2001.

[7] C. Monma, B. Munson, and W. Pulleyblank. Minimum-weight two-connected spanning networks. Mathematical Programming, 46:153-171, 1990. 